\title{
Endoscopic transpedicular thoracic discectomy
}

\section{Hae-Dong Jho, M.D., Ph.D.}

Department of Neurological Surgery, University of Pittsburgh School of Medicine, Pittsburgh, Pennsylvania

Object. To minimize the invasiveness and maximize the adequacy of the decompressive proceedure in thoracic discectomy, a $70^{\circ}$ endoscope was adopted to perform transpedicular thoracic discectomy.

Methods. A posterior transpedicular approach was performed via a 2-cm transverse skin incision, using the operating microscope or a $0^{\circ}$-lens endoscope. Using a $70^{\circ}$-lens endoscope, discectomy was performed after obtaining direct visualization of the ventral aspect of the spinal cord dura mater. This surgical technique has been used in 25 patients. There were 12 men and 13 women whose age ranged from 29 to 70 years (median 46 years). Thirteen patients experienced myelopathy, with or without radiculopathy, 10 presented with radiculopathy, and two patients suffered from segmental pain. The follow-up period ranged from 4 to 60 months (median 27 months). In 12 of the 13 patients with myelopathy, excellent improvement was shown postoperatively. In the remaining patient, symptoms recurred when she was injured in a motor vehicle accident 3 months postsurgery. In nine of 10 patients with radiculopathy pain resolved completely. In the one patient with right-sided hypochondral pain and in the two patients with segmental pain no relief was obtained despite excellent discectomy results that were demonstrated on postoperative magnetic resonance images. The average length of hospital stay was overnight.

Conclusions. The use of a $70^{\circ}$-lens endoscope via a transpedicular route has made thoracic discectomy comparable with cervical or lumbar discectomy in its surgical invasiveness, in the patient's recovery time, and in complexity of surgical procedure.

Key Words * endoscopy * intervertebral disc * minimally invasive neurosurgery * spine * thoracic discectomy * vertebra

Postoperative spinal cord damage had been a common result when treating thoracic disc herniation using the midline posterior laminectomy technique.[1] Over the past few decades, the surgical treatment of thoracic disc herniation via a posterior approach has changed from the traditional midline laminectomy to posterolateral approaches such as costotransversectomy, lateral extracavitary, and a transpedicular approach in an attempt to avoid catastrophic injury to the spinal cord.[2-7, 9] The surgical exposure via posterior approaches has been extended further laterally to provide a maximum ventral view of the spinal 
cord dura mater; however, this necessitated increased invasiveness. Among the various posterior surgical approaches, the least invasive is the transpedicular approach. With that technique, the surgical decompression of the ventral spinal cord must be performed blindly. Because of dissatisfaction with posterior approaches a transthoracic approach was developed that is more of an anterolateral than a completely anterior approach. Although the main advantage of this thoracotomy approach is the direct access to the anterior aspect of the spinal cord, the invasiveness of the surgical procedure itself is incomparable with that of cervical or lumbar disc surgery. Recently, an endoscopic transthoracic approach has been developed to minimize the invasiveness of the conventional thoracotomy technique.[8] Still, this endoscopic transthoracic technique requires that four separate skin incisions be made in the chest wall and that the patient undergo postoperative chest tube drainage for a few days.

In an attempt to maximize the benefit of the minimal invasiveness of posterior approaches, the use of a $70^{\circ}$ endoscope was adopted to visualize directly the ventral aspect of the spinal cord. Via a 2-cm-long transverse paramedian skin incision a transpedicular approach was performed. The medial portion of the facet joint and upper portion of the pedicle was removed to gain access to the protruded disc under the operating microscope or under a $0^{\circ}$-lens endoscope. A 4-mm-diameter rigid endoscope with a $70^{\circ}$ lens would then be mounted to a custom-made endoscope holder. Under the steady image of the endoscope, surgical decompression of the ventral spinal cord is performed using $90^{\circ}$-curved surgical instruments. The invasiveness of this transpedicular thoracic discectomy has been noted to be quite comparable with that of cervical or lumbar microdiscectomy.[3] In this report we include our experience with the use of an endoscope during transpedicular thoracic discectomy.

\section{CLINICAL MATERIAL AND METHODS}

\section{Patient Criteria}

Between November 1993 and December 1998, 25 patients who harbored thoracic disc protrusion underwent endoscopic transpedicular thoracic discectomy; all surgeries were performed by the author at the University of Pittsburgh Medical Center. This patient population included 12 men and 13 women, with ages ranging from 29 to 70 years (median 46 years). As their primary clinical symptom, seven patients suffered from myelopathy, six from radiculomyelopathy, 10 from radiculopathy, and two patients suffered from segmental pain. All patients underwent conservative therapy for more than 6 weeks, except for several of those suffering from myelopathy. In all patients magnetic resonance (MR) images of the thoracic spine were obtained. In some patients additional postmyelographic computerized tomography scanning was performed (Fig. 1 upper left and right). In all patients postoperative MR imaging of the thoracic spine was performed 6 weeks postoperatively (Fig. 1 lower left and right). Of the 13 patients with myelopathy, one patient experienced near-complete paraplegia for 36 hours, one patient had severe paraparesis that limited ambulation, and one patient suffered from severe spasticity that required the use of a cane to walk short distances. Of the 10 patients with radiculopathy, one patient was treated for hepatitis and experienced right-sided hypochondral pain. When her right hypochondral pain did not respond to medical treatment, an MR image of the thoracic spine was obtained that revealed a right-sided T11-12 disc protrusion. In the two patients with local segmental pain relief was not obtained by conservative treatments, and subsequent MR images demonstrated thoracic disc protrusion at the area corresponding with their pain. 


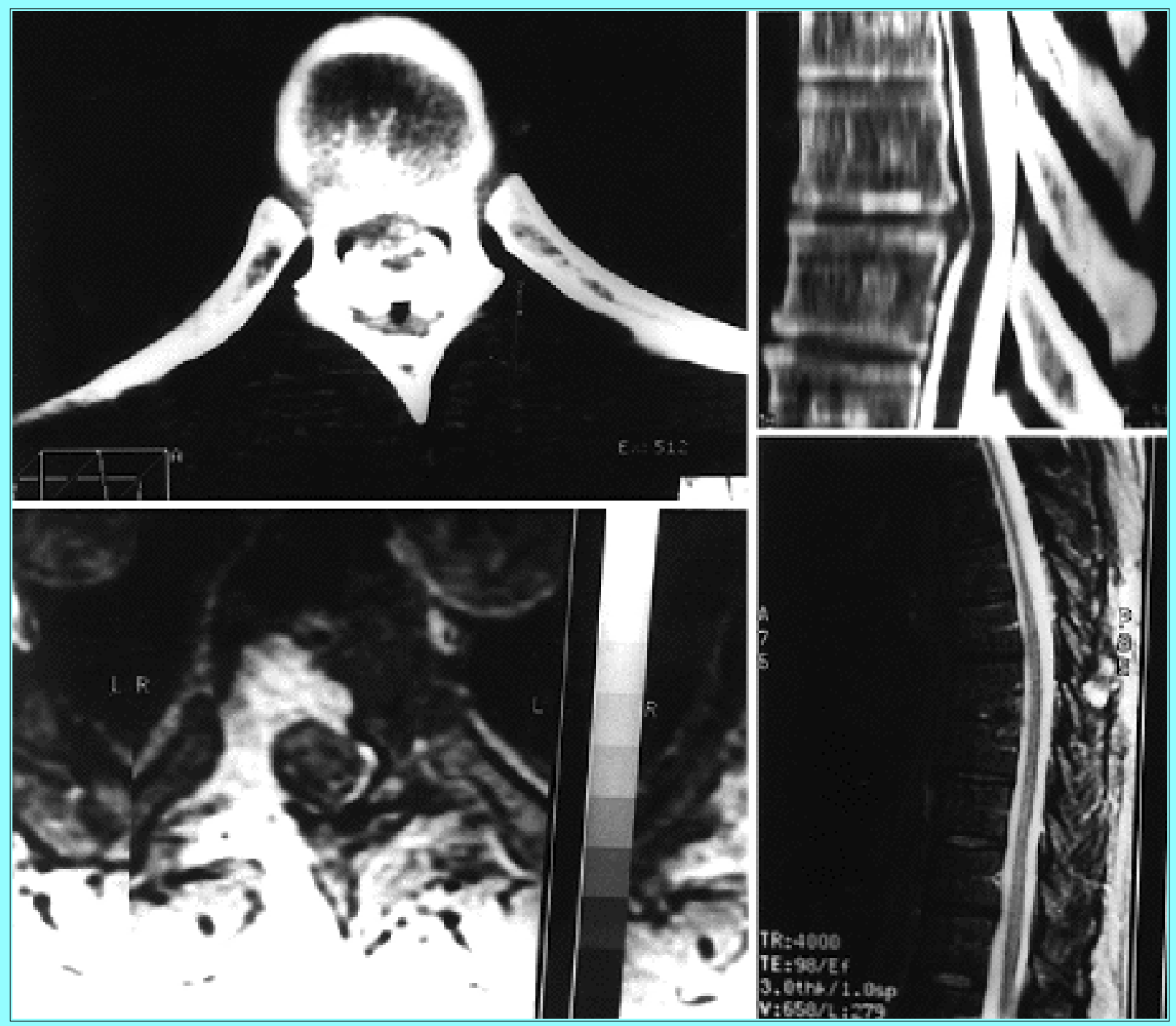

Fig. 1. Case 20. Postmyelographic computerized tomographic scans of the thoracic spine, axial (upper left) and sagittal views (upper right), revealing a T7-8 disc protrusion causing myelopathy. Postoperative MR images, axial (lower left) and sagittal views (lower right), showing complete removal of the protruded disc and excellent decompression of the spinal cord.

\section{Surgical Equipment}

We used a 4-mm-diameter and 18-cm-long rod lens rigid endoscope. In the transpedicular approach itself, a $0^{\circ}$-lens endoscope or operating microscope was used. Disc removal in front of the spinal cord was performed using a $70^{\circ}$ angled-lens endoscope. A custom-made endoscope holder is used to secure the endoscope tightly during the operation. A cleansing device is used for intermittent cleaning of the endoscope lens and is controlled by a foot pedal. A long-armed down-biting curette is used, which was custom made by bending the curette gently, close to $90^{\circ}$ at a point approximately $10 \mathrm{~mm}$ away from the curette tip. This curette tip can reach the contralateral side across the midline, ventral to the spinal cord. The tubular retractor is a $1.5-\mathrm{cm}$-diameter threaded trocar designed for endoscopic thoracotomy. In obese patients who require a longer tube, a 20 - or $30-\mathrm{mL}$ syringe barrel is used (the tip of the syringe barrel is cut to the appropriate length by using a pair of scissors or a rongeur).

\section{Operative Technique}

Details of the surgical technique have been previously reported.[3] General anesthesia is induced via 
endotracheal intubation. Somatosensory evoked potential monitoring is utilized during the entire procedure. The patient is positioned in a $60^{\circ}$ forwardly inclined lateral position that keeps the lesion-side up (Fig. 2). An axillary roll is placed under the dependent side to prevent injury to the neurovascular structures and to the shoulder joint. The correct thoracic spine level is corroborated using a portable radiography device. Preparation and draping are performed following completion of aseptic technique.

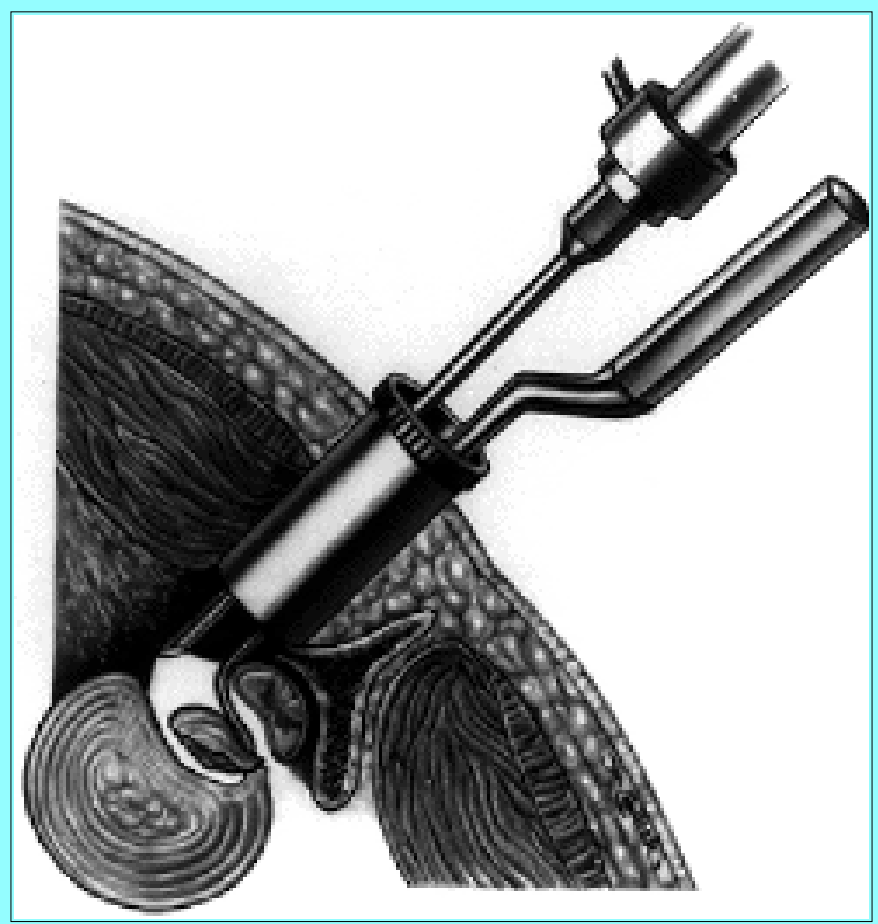

Fig. 2. Schematic drawing depicting the placement of a $1.5-\mathrm{cm}$ trocar retractor via a $2-\mathrm{cm}$ paramedian transverse skin incision, the application of the $70^{\circ}$-lens endoscope, and the use of right-angle down-biting curette for removal of the protruded disc ventral to the spinal cord. The patient is placed lesion-side up, in a $60^{\circ}$ forwardly inclined lateral position.

A 2-cm-long transverse skin incision is made laterally from the lateral margin of the corresponding spinous process. The paraspinal muscles are dissected from the spinous process, lamina, and transverse process by using a small periosteal elevator. A tubular trocar measuring $1.5 \mathrm{~cm}$ in diameter is introduced, exposing the facet and lamina. Under either a $0^{\circ}$-lens endoscope or operating microscope, the medial portion of the facet, the very lateral portion of the lamina, and the rostral one-third portion of the pedicle are removed using a high-speed drill. The very lateral margin of the spinal cord dura mater is exposed. The nerve root is usually identified rostral to the intervertebral disc, but it is occasionally encountered just above the resected portion of the pedicle. In cases of radiculopathy, the nerve root compression can be identified at this point. Using a high-speed drill at the area above and below the nerve root, the lateral bone spurs rostral and caudal to the herniated disc are removed, thus creating a small cavity. The drill bit is a 2-mm cutting bit. Limited exposure of the spinal cord dura mater will not permit the surgeon to injure the spinal cord because the main portion of the spinal cord is still covered by the remaining ipsilateral lamina. When an approximate $1.5-\mathrm{cm}$ cavity is created at the lateral intervertebral disc space by resection of the bone spur and disc material, the $70^{\circ}$-lens endoscope is used.

As described previously, the $70^{\circ}$-lens endoscope is mounted to the endoscope holder. The endoscope is used to visualize the ventral aspect of the spinal cord dura mater directly. Under this direct endoscopic visualization, the cavity that was created lateral to the spinal cord is extended further medially by removing more disc material and bone spurs with the down-biting curette. When an extended tunnel has 
been created beneath a thin layer of the dorsally protruded disc and bone spur that have been compressing the spinal cord, the shell of the disc material and bone spur beneath the spinal cord are pushed away from the spinal cord toward the cavity and then removed using a long-armed down-biting curette and a curved pituitary rongeur. To perform surgery with the $70^{\circ}$ endoscope does require practice. When adequate surgical decompression has been performed, the ventrally concave curvature of the spinal cord dura mater bulges out to form a convex curvature ventrally toward the intervertebral space (Fig. 3). Bone bleeding is controlled with application of bone wax. The trocar retractor is removed. A couple of stitches are placed at the paraspinal fascia. A local anesthetic is administered to minimize postoperative incisional pain. Subcuticular skin closure is performed, and a small bandage is applied. This transpedicular thoracic discectomy for the treatment of radiculopathy had been performed on an outpatient basis. The patients with myelopathy were kept overnight in the hospital for observation.

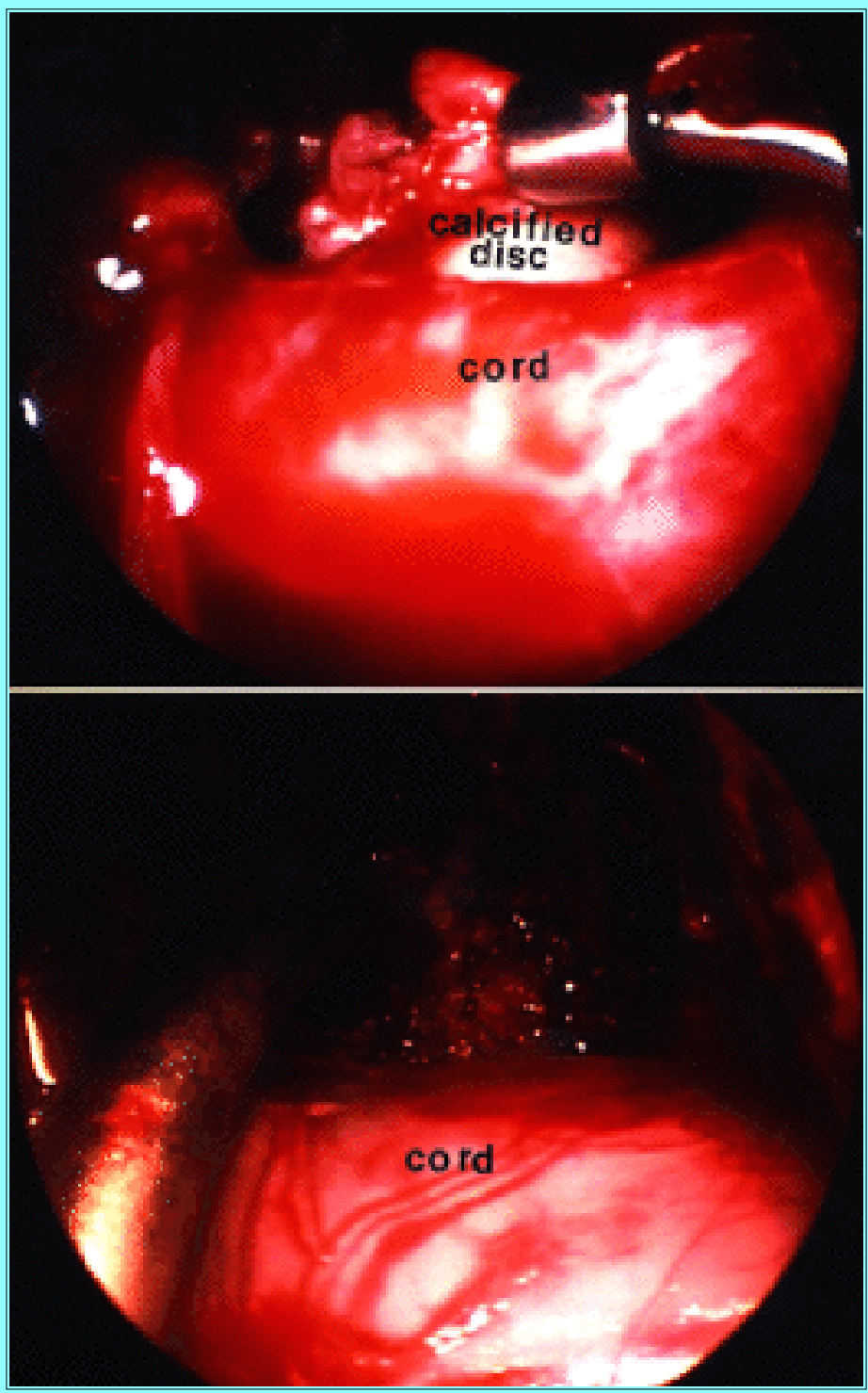

Fig. 3. Intraoperative photographs. Upper: View obtained with $70^{\circ}$-lens endoscope revealing a protruded calcified disc that is indentating the ventral aspect of the spinal cord. Lower: View obtained after removal of protruded disc showing the ventral aspect of the spinal cord dura mater bulging out toward the cavity created at the intervertebral disc space.

\section{RESULTS}

Table 1 provides a summary of patient outcomes. No surgery-related complications were encountered in 
this series of patients. Of 13 patients with myelopathy, with or without radicular pain, excellent postoperative improvement of symptoms was shown in 12 patients. One patient (Case 22; Table 1) with almost flaccid, complete paraplegia recovered normal function postoperatively. Surprisingly, he did not experience any residual symptoms of myelopathy. His reflexes returned to normal after surgery. Another patient (Case 5) with profound preoperative paraparesis was able to walk without assistance postoperatively. In one patient (Case 17) who was unable to ambulate preoperatively, ambulatory status improved and the patient now walks with a cane. He still experiences residual spasticity. One patient (Case 13) with radiculomyelopathy experienced recurrence of symptoms after a motor vehicle accident 3 months postoperatively. Subsequently she underwent two more operations by other surgeons (endoscopic transthoracic discectomy and conventional transthoracic discectomy, respectively) at the same level of the thoracic disc. Despite multiple operations, her right-sided hypochondral pain was not relieved, and she remained hyperreflexic. The remaining patients with myelopathy made complete recoveries. Of the 10 patients with radiculopathy, in nine relief from their radicular pain was obtained. One patient (Case 15), who had undergone multiple abdominal treatments (such as cholecystectomy and pancreatic stent placement) for right-sided hypochondral pain, was found to have a right-sided T11-12 disc protrusion. She underwent a right-sided approach for T11-12 transpedicular discectomy; however, she continued to experienced right-sided hypochondral pain postoperatively. In two patients with local segmental pain at the corresponding level of their thoracic disc protrusions the pain was not relieved, despite excellent removal of the protruded disc, which was demonstrated on postoperative MR imaging.

\begin{tabular}{|c|c|c|c|c|c|}
\hline & ICAL AND O & $\begin{array}{l}\text { ME DATA } \\
\text { ENDOS }\end{array}$ & $\begin{array}{l}\text { TABLE } 1 \\
\text { PATIENTS WHO UNDEF } \\
\text { THORAC K DISCECTOM }\end{array}$ & IT TRANSF & ICULAR \\
\hline $\begin{array}{l}\text { Case } \\
\text { No. }\end{array}$ & $\begin{array}{c}\text { Age }(y s), \\
\text { \& Sex }\end{array}$ & Level & $\begin{array}{l}\text { Presenting } \\
\text { Symptoms }\end{array}$ & $\begin{array}{c}\text { Follow } \\
\text { Up (Mos) }\end{array}$ & Outcome \\
\hline 1 & $44, M$ & T8-9 & radiculom yelopathy & 60 & excellent \\
\hline 2 & $44, F$ & $T 7-8$ & m yelopathy & 58 & excellent \\
\hline 3 & 40, M & $\mathrm{T} 11-12$ & radiculo pathy & 53 & excellent \\
\hline 4 & $45, F$ & $T 5-7$ & segmental pain & 47 & no relief \\
\hline 5 & $70, \mathrm{~F}$ & $T 1-2$ & m yelopathy & 46 & im pro ied \\
\hline 6 & $61, F$ & $T-8$ & $m$ yelopathy & 45 & excellent \\
\hline 7 & $52, \mathrm{~F}$ & T10-11 & radiculo pathy & 35 & excellent \\
\hline 8 & $31, F$ & $T 9-10$ & radiculom yelopathy & 34 & excellent \\
\hline 9 & 61 , M & $T \rightarrow 8$ & radiculo pathy & 30 & excellent \\
\hline 10 & $53, F$ & T8-9 & radiculo pathy & 30 & excellent \\
\hline 11 & 74 , M & $T 5-6$ & m yelopathy & 30 & excellent \\
\hline 12 & 53 , M & $T 9-10$ & radiculom yelopathy & 28 & excellent \\
\hline 13 & $36, F$ & $T 11-12$ & radiculom yelopathy & 27 & recurred \\
\hline 14 & $43 \mathrm{~F}$ & $\mathrm{~T} 10-11$ & radiculo pathy & 25 & excellent \\
\hline 15 & $39, \mathrm{~F}$ & $\mathrm{~T} 11-12$ & hypochondral pain & 25 & no relief \\
\hline 16 & $54, \mathrm{~F}$ & T8-9 & radiculo pathy & 22 & excellent \\
\hline 17 & 69 M & $75-6$ & m yelopathy & 21 & im pro ied \\
\hline 18 & 52 , M & $T 7-8$ & radiculo pathy & 18 & excellent \\
\hline 19 & 49 , w & $\mathrm{T} 11-12$ & radiculo pathy & 14 & excellent \\
\hline 20 & 44 , M & $T 7-8$ & m yelopathy & 11 & im proved \\
\hline 21 & $31, \mathrm{~F}$ & $\pi / 8$ & segmental pain & 9 & no relief \\
\hline 22 & $29 \mathrm{M}$ & $\mathrm{T} 6-7$ & near paraplegia & 9 & excellent \\
\hline 23 & 46 , M & $T 7-8$ & radiculom yelopathy & 6 & excellent \\
\hline 24 & $50, F$ & $T \gamma-9$ & radiculo pathy & 4 & excellent \\
\hline 25 & $45, M$ & $\mathrm{~T} 6-7$ & radiculo pathy & 4 & excellent \\
\hline
\end{tabular}


The transpedicular approach to thoracic discs was first introduced by Patterson and Arbit[6] in 1978, and the surgical procedure adopted by the author in this series is very similar to their original description.[3] Stillerman, et al.,[9] reported a similar surgical technique and named their approach the "transfacet pedicle-sparing approach." The intervertebral disc in the thoracic spine is located at the level of the facet joint. To expose the intervertebral disc laterally to the spinal cord, the facet joint has to be removed either partially or totally. In addition, the rostral edge of the pedicle is so close to the intervertebral disc in the thoracic spine that the rostral one-third portion of the pedicle at the lower vertebra has to be removed to obtain adequate exposure of the bone edge of the lower vertebra adjacent to the intervertebral disc. In this procedure, the key structures to be removed (to access a thoracic intervertebral disc via a posterior paramedian approach) are a medial portion of the facet joint and the rostral one-third of the pedicle. Coining another name for this surgical technique, as these authors have done, may only add confusion; however, referring to this as a "posterior paramedian" approach might be more of an accurate description than naming it a "transfacet" or "transpedicular" approach. From skin incision to placement of the trocar retractor, the surgical procedure is fast, lasting only a few minutes, and straight forward. The very lateral portion of the laminae and the medial portion of the facet joint is removed using a high-speed drill with a 2-mm cutting bit. The surgical landmark is the crossing point of a line drawn along the inferior margin of the facet process horizontally and a vertical line drawn along the midpoint of the facet joint. The crossing point of these two lines will lead to the rostral portion of the pedicle. Other landmarks are the transverse processes of adjacent vertebrae. The facet joint can be identified just medially in between the transverse processes. Drilling of the bone can be performed under visualization of the operating microscope or the $0^{\circ}$-lens endoscope. Use of the operating microscope has enabled more controlled and safe drilling than use of the endoscope, mostly because of the stereoscopic view provided by the former. When a three-dimensional endoscope is adopted with a high-definition video monitor, endoscopic stereoscopic images might become comparable with the views provided by the operating microscope. As Patterson and Arbit[6] originally reported, it is important to create a small cavity lateral to the spinal cord first, by removing the bone spur of the rostral and caudal vertebra in addition to the removal of the intervertebral disc before any attempt at removal of the disc is made medially.

Operating under the $70^{\circ}$-lens endoscope is very difficult and confusing for a beginner. With this surgical technique, the very edge of the lateral portion of the spinal cord dura mater is exposed, roughly 2 to 3 $\mathrm{mm}$. The remaining lamina is still covers most of the spinal cord. This limited exposure of the spinal cord helps to avoid inadvertent damage to the spinal cord when surgical instruments are passed in or out of the visual field provided by a $70^{\circ}$-lens endoscope. In addition to the confusing view of $70^{\circ}$-lens endoscope, the surgeon's use of curved surgical instruments can become a kind of double jeopardy until he or she becomes familiar with the technique. An endoscope holder is essential to maintain a steady image on the monitor, and it also allows a surgeon to use both hands. An endoscope cleaning device is another integral element in this endoscopic technique. Although the currently available commercial endoscope cleaner is not yet ideal, it still helps the surgeon to operate continuously without frequent interruption to cleanse a foggy or blood-stained lens. Surgical instruments have to be curved to be introduced under the spinal cord.

Recently, endoscopic transthoracic discectomy has been introduced.[8] This transthoracic endoscopic technique still requires that three to four separate skin incisions be made, as well as postoperative chest tube drainage for a few days. Although a transthoracic endoscopic approach to a thoracic disc is relatively minimally invasive when compared with the conventional thoracotomy technique, it is still more demanding and invasive when compared with other lumbar or cervical disc operations. Other 
authors have described their occasional use of endoscopes to inspect the ventral aspect of the spinal cord dura mater during a posterior approach to thoracic disc protrusions.[5,9] The use of an angled-lens endoscope can be broadened to include various posterior approaches to the spine at any level of the vertebrae, and its use may minimize unnecessary extensive surgical exploration, which is often required for exposure at the ventral aspect of the spinal cord.

In the current series of patients, in the two patients with local segmental pain no pain relief was obtained, despite the adequate removal of the protruded disc at the corresponding level. Both patients had sustained work-related injuries and developed local midline pain at the thoracic spine, which did not respond to prolonged conservative therapy. Preoperative MR images revealed disc protrusion at the vertebral level that corresponded with their pain. Postoperatively, both patients continued to experience pain. One patient with right-sided hypochondral pain and hepatitis was diagnosed as having a T11-12 disc protrusion based on MR imaging evaluation. Despite successful removal of the protruded disc, this patient continued to experience right-sided hypochondral pain. The other patient (Case 13) with right-sided hypochondral pain had undergone multiple abdominal procedures and was diagnosed as having a T11-12 disc protrusion. In this patient relatively increased reflexes in the lower extremities were demonstrated. Endoscopic discectomy seemed to relieve symptoms for 3 months until she sustained an injury in a motor vehicle accident. Her symptoms fully recurred. Subsequently, she underwent endoscopic transthoracic discectomy and fusion procedures, which were performed by other surgeons, with no relief of her symptoms. She underwent a third procedure, a conventional thoracotomy approach performed by other surgeons, with no relief of her symptoms. The hypochondral pain that these two patients experienced might not have been caused by thoracic disc protrusion. Both had undergone prolonged and various therapy to treat their abdominal disorders preoperatively.

Although no patient in this series developed surgery-related spinal cord damage, it remains the main potential risk of this endoscopic transpedicular technique. Although somatosensory evoked potential monitoring was used in all patients, its use does not preclude the development of intraoperative spinal cord injury. Once a surgeon decides to use an endoscope in a posterior approach to thoracic discs, a posterolateral extracavitary approach may be an easy operation with which to start. With this wider and further lateral exposure, a surgeon may adapt to the use of a $70^{\circ}$-lens endoscope a bit more easily. When practice has improved familiarity with and adroitness in endoscope use, a more restricted surgical exposure, such as a transpedicular approach, can be performed. Endoscopic inspection of the ventral aspect of the spinal cord alone is not especially difficult. When surgical decompression of the spinal cord is achieved, the spinal cord dura mater bulges out toward the site of the discectomy cavity, showing convexity on the endoscopic view. The absence of this dural bulging indicates that there has been inadequate decompression of the spinal cord, which is another potential unwanted outcome. Nerve root damage likewise is a possible complication when a conventional $0^{\circ}$-lens endoscope is used for the surgical exposure. The lack of depth perception in the monitored two-dimensional image may lead to injury to the nerve root while the facet joint is removed. However, no single surgery-related complication has been encountered in the author's series of patients. When the surgeon becomes familiar with the surgical technique, the patient's postoperative recovery, the amount of surgical invasiveness, and the complexity of the surgical procedure may become comparable with those associated with cervical or lumbar discectomy.

\section{Acknowledgments}

The author wishes to thank Arthur P. Nestler, B.S.N., and Robin Coret, B.A., for their assistance with 
preparation of the manuscript.

\section{References}

1. Arseni C, Nash F: Thoracic intervertebral disc protrusion. A clinical study. J Neurosurg 17:418-430, 1960

2. Hulme A: The surgical approach to thoracic intervertebral disc protrusions. J Neurol Neurosurg Psychiatry 23:133-137, 1960

3. Jho HD: Endoscopic microscopic transpedicular thoracic discectomy. Technical note. J Neurosurg 87:125-129, 1997

4. Larson SJ, Holst RA, Hemmy DC, et al: Lateral extracavitary approach to traumatic lesions of the thoracic and lumbar spine. J Neurosurg 45:628-637, 1976

5. Le Roux PD, Haglund MM, Hemmy DC, et al: Thoracic disc disease: experience with the transpedicular approach in twenty consecutive patients. Neurosurgery 33:58-66, 1993

6. Patterson RH Jr, Arbit E: A surgical approach through the pedicle to protruded thoracic discs. J Neurosurg 48:768-772, 1978

7. Perot PL Jr, Munro DD: Transthoracic removal of midline thoracic disc protrusions causing spinal cord compression. J Neurosurg 31:452-458, 1969

8. Rosenthal D, Rosenthal R, de Simone A: Removal of a protruded thoracic disc using microsurgical endoscopy. A new technique. Spine 19:1087-1091, 1994

9. Stillerman CB, Chen TC, Diaz Day J, et al: The transfacet pedicle-sparing approach for thoracic disc removal: cadaveric morphometric analysis and preliminary clinical experience. J Neurosurg 83:971-976, 1995

Manuscript received March 16, 1999.

Accepted in final form April 28, 1999.

Address reprint requests to: Hae-Dong Jho, M.D., Ph.D., Department of Neurological Surgery, Presbyterian University Hospital, Suite B-400, 200 Lothrop Street, Pittsburgh, Pennsylvania 15213. email: hdjho@neuronet.pitt.edu. 